\title{
Nigrostriatal Lesions Alter Oral Dyskinesia and c-Fos Expression Induced by the Serotonin Agonist 1-(m-Chlorophenyl)piperazine in Adult Rats
}

\author{
Philippe De Deurwaerdère and Marie-Françoise Chesselet \\ Department of Neurology, University of California, Los Angeles School of Medicine, Los Angeles, California 90095
}

The loss of dopaminergic innervation of the basal ganglia, a group of subcortical regions involved in motor control, is the hallmark of Parkinson's disease. The resulting molecular and cellular alterations mediate behavioral deficits and may modify neuronal responses to other neurotransmitters. In the present study, we sought to determine the effects of chronic dopamine (DA) depletion on responses mediated by stimulation of serotonergic $2 \mathrm{C}\left(5-\mathrm{HT}_{2 \mathrm{C}}\right)$ receptors, a serotonergic receptor subtype present in discrete regions of the basal ganglia. Specifically, the effects of unilateral lesions of nigrostriatal DA neurons on oral dyskinesia and Fos protein expression induced by the non-selective $5-\mathrm{HT}_{2 \mathrm{C}}$ agonist 1 -( $m$-chlorophenyl)piperazine ( $m$ CPP) were examined.

Confirming previous findings, both peripheral and local injections of $m$-CPP into the subthalamic nucleus elicited oral dys-

Parkinson's disease is characterized by the progressive loss of nigrostriatal dopaminergic neurons. Numerous studies have demonstrated long-term consequences of dopamine (DA) depletion on GABAergic and glutamatergic functions in the basal ganglia (Chesselet, 2000). However, much less is known about the effects of nigrostriatal lesions on serotonergic function.

Serotonergic neurons innervate all regions of the basal ganglia and act on several receptor subtypes (Azmitia and Segal, 1978; Barnes and Sharp, 1999). Serotonergic $2 \mathrm{C}\left(5-\mathrm{HT}_{2 \mathrm{C}}\right)$ receptors are of particular interest because they are known to mediate oral dyskinesia (Stewart et al., 1989; Gong et al., 1992; Eberle-Wang et al., 1996), a side effect of prolonged administration of either dopamine antagonists or L-3,4-dihydroxyphenylalanine (LDOPA), the main treatment of Parkinson's disease (Tarsy and Baldessarini, 1984; Chase et al., 1994; Ebabi and Srinivasan, 1995). Indeed preclinical and clinical studies suggest that increased serotonin (5-HT) transmission plays a role in dyskinesia (Meltzer and Nash, 1991; Melamed et al., 1996) and that drug acting at 5- $\mathrm{HT}_{2 \mathrm{C}}$ receptors may be useful in Parkinson's disease and schizophrenia (Roth et al., 1992; Ikeguchi and Kuroda, 1995; Fox et al., 1998).

$5-\mathrm{HT}_{2 \mathrm{C}}$ receptors are present in the striatum, the substantia nigra pars reticulata $(\mathrm{SNr})$, the internal segment of the globus pallidus (GP) [entopeduncular nucleus (EPN) in rats], and the

Received Feb. 22, 2000; revised April 12, 2000; accepted April 18, 2000.

This work was supported by Public Health Service Grants R37-MH44894 and P50-NS 38367. We thank Cynthia Ho. for her help with some experiments.

Correspondence should be addressed to Dr. Marie-Francoise Chesselet, Department of Neurology, University of California, Los Angeles School of Medicine, 710 Westwood Plaza, Los Angeles, CA 90095-1769. E-mail: mchessel@ucla.edu. Copyright (C) 2000 Society for Neuroscience $0270-6474 / 00 / 205170-09 \$ 15.00 / 0$ kinesia. Nigrostriatal lesions markedly enhanced oral bouts induced by peripheral but not intrasubthalamic administration of $m$-CPP. In intact rats, Fos expression was increased by $m$-CPP (1 $\mathrm{mg} / \mathrm{kg}$, i.p.) in the striatum and the subthalamic nucleus. After nigrostriatal lesions, $m$-CPP-induced Fos expression remained unchanged in the subthalamic nucleus but was reduced in the medial quadrants of the striatum and was markedly enhanced in the entopeduncular nucleus. These data demonstrate regionally specific alterations in behavioral and cellular responses to a serotonergic agonist in an animal model of Parkinson's disease.

Key words: Parkinson's disease; dyskinesia; dopamine; serotonin; basal ganglia; serotonin $2 \mathrm{C}$ receptors; c-Fos; $\mathrm{m}-\mathrm{CPP}$

subthalamic nucleus (Pazos et al., 1985; Eberle-Wang et al., 1997; Pasqualetti et al., 1999; Clemett et al., 2000). Evidence indicates that $5-\mathrm{HT}_{2 \mathrm{C}}$ receptors contribute to the regulation of dopaminergic neurons (Trent and Tepper, 1991; De Deurwaerdère and Spampinato, 1999). However, dopaminergic neurons do not express 5- $\mathrm{HT}_{2 \mathrm{C}}$ receptor mRNA (Eberle-Wang et al., 1997; Pasqualetti et al., 1999), suggesting that this effect is indirect. In contrast, electrophysiological, biochemical, and behavioral data showed a direct influence of $5-\mathrm{HT}_{2 \mathrm{C}}$ receptors on GABAergic neurons of the striatum and the substantia nigra pars reticulata (El Mansari et al., 1994; Rick et al., 1995; Fox et al., 1998) and on glutamatergic neurons of the subthalamic nucleus (Eberle-Wang et al., 1996).

To understand the functional consequences of the loss of dopaminergic neurons, as occurs in Parkinson's disease, it is important to elucidate how chronic alterations in dopamine levels alter neuronal responses to remaining neurotransmitters. Behavioral experiments have revealed that lesions of dopaminergic neurons in neonates dramatically increase the response to the nonselective $5-\mathrm{HT}_{2 \mathrm{C}}$ agonist 1 - $(m$-chlorophenyl)piperazine $(\mathrm{m}$ CPP) in adult rats (Gong and Kostrzewa, 1992). However, it is unclear whether this potentiation is the consequence of a supersensitivity to 5-HT agonists occurring only in rats with a neonatal lesion (El Mansari et al., 1994; Kostrzewa et al., 1998) or whether behavioral or cellular responses to $m$-CPP are also altered by nigrostriatal lesions in adults.

In the present study, we sought to determine whether a unilateral lesion of the nigrostriatal dopaminergic pathway in adult rats, a model of Parkinson's disease, alters the ability of $m$-CPP to (1) induce oral movements in rats and (2) induce the expression of 
Fos protein, the product of the immediate early gene c-fos, a tool for exploring neuronal responses in discrete brain regions.

\section{MATERIALS AND METHODS}

Surgeries. All procedures were performed on male Sprague Dawley rats (Charles River Laboratories, Los Angeles, CA) in accordance with the National Institutes of Health Guide for the Care and Use of Laboratory Animals and were approved by the local animal care committee. Before any surgery, rats (250-275 gm) were maintained on a $12 \mathrm{hr}$ light/dark schedule with food and water available ad libitum.

Lesions of the dopaminergic neurons located in the substantia nigra pars compacta $(\mathrm{SNc})$ were performed as described previously (Delfs et al., 1995). Briefly, rats were anesthetized with equithesin (prepared as per the instruction of Janssen-Salbutry Laboratories, Kansas City, MO) and placed in a stereotaxic frame (David Kopf Instruments, Tujunga, $\mathrm{CA})$. The tip of the injector was stereotaxically implanted in the left substantia nigra pars compacta [anteroposterior (AP), 3.4; lateral (L), 1.8; ventral (V), 2 with respect to interaural zero] (Paxinos and Watson, 1986). Eight micrograms (free base) of 6-hydroxydopamine (6-OHDA) (Sigma, St. Louis, MO), dissolved in $4 \mu \mathrm{l}$ of $\mathrm{NaCl}$ containing $0.1 \%$ ascorbic acid, were administered through the cannula over $4.50 \mathrm{~min}$, and the cannula was left in place for 5 min after the injection before being slowly removed. Rats were pretreated $30 \mathrm{~min}$ before the 6-OHDA infusion with $25 \mathrm{mg} / \mathrm{kg}$ desipramine to protect noradrenergic projections. Rats given "sham" lesions received an injection of desipramine, followed by vehicle only into the substantia nigra.

Three weeks after infusion of 6-OHDA or its vehicle in the SNc, rats were screened for the completeness of the lesion by subcutaneous injection of $0.05 \mathrm{mg} / \mathrm{kg}$ apomorphine. Rats with nigrostriatal lesions that did not show at least 100 rotations during 30 min after apomorphine injection were excluded from the study. Sham-lesioned rats also received the apomorphine injection but, as expected, did not show any rotational behavior.

In experiments requiring local infusion into the subthalamic nucleus, rats were anesthetized with equithesin 2 weeks after the intranigral administration of 6-OHDA or its vehicle and placed in a stereotaxic frame. The guide cannula (22 gauge inner diameter; Plastics One Inc., Roanoke, VA) was stereotaxically positioned such that the tip of the injector was located at the following coordinates: AP, 5.1; L, 2; V, 2.1 corresponding to the left subthalamic nucleus relative to the interaural zero (Paxinos and Watson, 1986). The guide shaft was secured to the skull with dental resin (SNAP; Parkell, Farmingdale, NY) and anchored to stainless steel mounting screws placed in the skull. After this surgery, rats were housed individually.

Injectors used during the course of this study were constructed from commercially available dummy and internal cannulas (Plastics One Inc.), as described previously (Parry et al., 1994). Briefly, an injector was prepared by threading a $35 \mathrm{~cm}$ piece of fused silica (outer diameter of 150 $\mu \mathrm{m}$; inner diameter of $73 \mu \mathrm{m}$ ) through a 28 gauge internal cannula such that a $1 \mathrm{~mm}$ tip of fused silica extended from the end of the cannula shaft. The fused silica portion of the injector was sheathed up to the internal cannula in polyethylene-50 tubing, and all junctions were secured with epoxy.

Behavioral testing. Rats were exposed to the behavioral room conditions before the testing day to facilitate their adaptation to transportation and to the behavioral room. On the day of behavioral testing, each rat was placed in clear circular plastic chambers (12 inches in diameter; 18 inches in height). After a $1 \mathrm{hr}$ adaptation period and immediately before the onset of behavioral observations, the 5-HT agonist $m$-CPP or its vehicle were administered either systemically (intraperitoneally) or locally into the subthalamic nucleus.

Details of the procedure for microinfusion into the subthalamic nucleus have been described in previous reports (Parry et al., 1994). The awake rats were gently hand-held, and $m$-CPP or its vehicle were infused directly into the subthalamic nucleus via an injector placed within the surgically implanted guide cannula. A total volume of $0.1 \mu \mathrm{l}$ was inf used over $54 \mathrm{sec}$. The injector was left in place for an additional $60 \mathrm{sec}$ to allow diff usion of the drug away from the injector tip.

After either type of drug injection, the rats were returned to the testing cage and observed continuously for $60 \mathrm{~min}$ for bouts of orofacial movements. The number of oral bouts/60 min was quantified by an observer blind to the drug schedule administration. Oral activity was defined as vacuous chewing, jaw tremors, and tongue darting that occurred without any reference to an evident physical material. Thus, oral activity occurring with feeding, grooming, licking, or sniffing was not counted. More- over, an interval of $2 \mathrm{sec}$ without oral activity was required to consider the oral bout fully terminated. The duration of each oral bout was not measured (Parry et al., 1994; Eberle-Wang et al., 1996; Mehta et al., 2000).

Pharmacological treatment. All experiments assessing the effect of $m$-CPP on oral movements or on Fos expression started 4.5 weeks after the lesion of DA neurons and were done during the diurnal portion of the day. In the case of systemic injection, rats with sham or 6-OHDA lesions were given only one injection ( $m$-CPP or saline). After the $1 \mathrm{hr}$ period of behavioral testing, rats were returned to their cages. Two hours after $m$-CPP or saline injection, rats were anesthetized and perfused with $4 \%$ paraformaldehyde solution (see below) for Fos and tyrosine hydroxylase (TH) immunohistochemistry.

In the case of local administration of $m$-CPP into the subthalamic nucleus, doses of $m$-CPP $(0,0.2,0.5$, and $1 \mu \mathrm{g} / 100 \mathrm{nl})$ were injected in a counterbalanced order over a 4 week period (1 week interval between injections), such that, at the completion of testing, most rats had received all doses of $m$-CPP in a random order. Some rats (three of eight rats in each of sham and 6-OHDA-treated groups) lost their head block during the course of the study and, consequently, did not receive all doses. Considering the random design of the pharmacological treatment, results from these rats were included. At the end of the entire experiment, rats were anesthetized and perfused for TH immunohistochemistry experiments and verification of cannula placement into the subthalamic nucleus.

Immunohistochemistry. Rats were deeply anesthetized with equithesin and perfused transcardially with $0.1 \mathrm{M} \mathrm{PBS}, \mathrm{pH} 7.4$ (at $37^{\circ} \mathrm{C}$ ), followed by ice-cold $4 \%$ paraformaldehyde in $0.1 \mathrm{~m}$ sodium phosphate buffer (PB). Brains were removed, post-fixed for $2 \mathrm{hr}$ in the same fixative, and sunk in $30 \%$ sucrose at $4^{\circ} \mathrm{C}$. Brains were then frozen in cold isopentane (approximately $-35^{\circ} \mathrm{C}$ ) and stored at $-80^{\circ} \mathrm{C}$. Forebrains were cut at $40 \mu \mathrm{m}$ on a cryostat (CM1800; Leica, Nussloch, Germany) in the coronal plane. Sections through the striatum, GP, EPN, subthalamic nucleus, and substantia nigra were collected in PBS $\left(0.1 \mathrm{M}, 4^{\circ} \mathrm{C}\right)$, placed in cold cryoprotecting solution containing phosphate buffer $(0.05 \mathrm{~mm})$, glycerol $(50 \%$ $\mathrm{v} / \mathrm{v})$, sucrose $(8 \%)$, and $\mathrm{MgCl}_{2}(1 \mathrm{~mm})$, and stored at $-24^{\circ} \mathrm{C}$ pending Fos or TH immunohistochemistry. A Nissl staining was performed on adjacent sections for identification of structures.

After four washes in $0.1 \mathrm{M} \mathrm{PB}, \mathrm{pH} 7.4$, at room temperature, sections were incubated in 5\% normal goat serum with $0.2 \%$ Triton X-100 and then transferred to $1 \%$ normal goat serum containing anti-Fos rabbit polyclonal antibody (1:20,000; PC38; Oncogene Science, Cambridge, $\mathrm{MA}$ ) for $40 \mathrm{hr}$ at $4^{\circ} \mathrm{C}$. After six washes in $0.1 \mathrm{M} \mathrm{PB}$ (5 min each), sections were incubated during $1 \mathrm{hr}$ in $0.1 \mathrm{M} \mathrm{PB}$ containing $1 \%$ normal goat serum and biotinylated goat anti-rabbit IgG (1:200; Vector Laboratories, Burlingame, CA). After washes (three times in $0.1 \mathrm{M} \mathrm{PB}$ ), sections were incubated in avidin-biotin-peroxidase complex (1 hr; Vector Laboratories) for subsequent staining with 3,3'-diaminobenzidine $(0.05 \% \mathrm{w} / \mathrm{v}$; Sigma) in $0.1 \mathrm{M}$ PB containing $0.003 \% \mathrm{H}_{2} \mathrm{O}_{2}$ (Sigma). Sections were finally washed three times in $0.1 \mathrm{M}$ Tris buffer, $\mathrm{pH}$ 7.4. The specificity of the immunostaining was assessed by omission of the primary or the secondary antibody from the protocol. After processing, tissue sections were mounted onto gelatin-alum-coated slides, dehydrated in ascending concentration of ethanol, and coverslipped with Eukitt mounting medium (Calibrated Instruments, Hawthorne, NY).

For TH immunolabeling, $40-\mu \mathrm{m}$-thick midbrain sections were incubated in 5\% normal goat serum with $0.3 \%$ Triton X-100 in $0.1 \mathrm{M}$ PBS and then transferred to $2 \%$ normal goat serum containing anti-TH rabbit polyclonal serum (1:5000; AB151; Chemicon, Temecula, CA) for $72 \mathrm{hr}$ at $4^{\circ} \mathrm{C}$. After four washes in $0.1 \mathrm{M}$ PBS $(10 \mathrm{~min}$ each $)$, sections were incubated during $1 \mathrm{hr}$ in $0.1 \mathrm{M}$ PBS containing $2 \%$ normal goat serum and biotinylated goat anti-rabbit IgG (1:200, Vector Laboratories Inc., Burlingame, CA). Subsequent steps were similar to that described above for Fos immunolabeling.

Verification of cannula placement. Fifty-micrometer-thick sections were cut through the subthalamic nucleus and stained with cresyl violet for verification of cannula placement within the subthalamic nucleus. Only data from rats in which accurate cannula tract was verified were retained for analysis. Representative tissue sections depicting cannula placement have been published previously (Parry et al., 1994; Eberle-Wang et al., 1996).

Data analysis. Before quantitative analysis, sections were viewed at a low magnification, and anatomical regions were outlined based on Nissl staining in serially adjacent sections. Two sections per region were analyzed, except for the $\mathrm{SNr}$ in which four sections were analyzed. The 


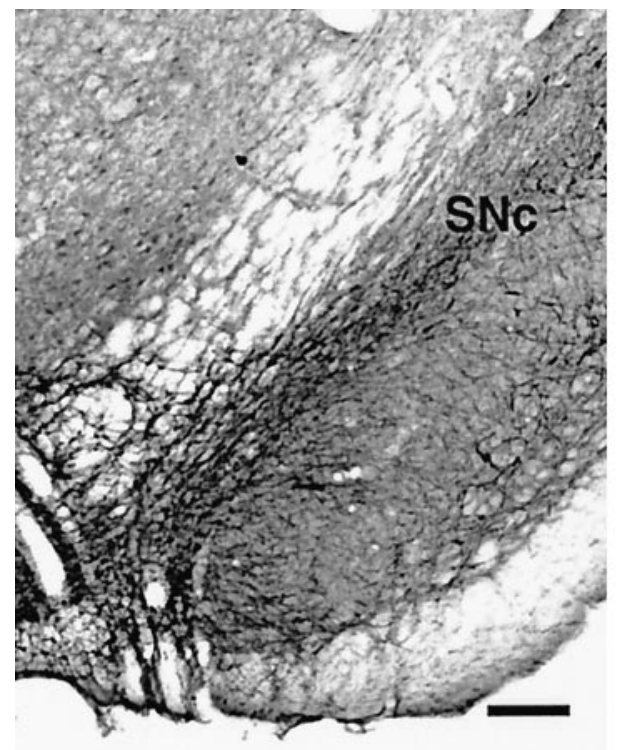

Figure 1. Tyrosine hydroxylase immunoreactivity in the substantia nigra of shamtreated (left) and 6-OHDA-treated (right) rats. Photomicrographs show the absence of dopaminergic neurons on the lesion side in rats treated with 6-OHDA. Dopaminergic neurons are preserved in the ventral tegmental area $(V T A)$. Scale bar, $200 \mu \mathrm{m}$. total surface area tissue area examined in this way did not differ between groups and was approximately as follow: subthalamic nucleus, $0.17 \mathrm{~mm}^{2}$; $\mathrm{SNr}, 0.7 \mathrm{~mm}^{2}$; EPN, $0.25 \mathrm{~mm}^{2}$; GP, $0.3 \mathrm{~mm}^{2}$. As reported previously (Rouillard et al., 1996), the striatum has been analyzed in four quadrants $\left(\sim 0.5 \mathrm{~mm}^{2}\right.$ each) corresponding to dorsomedial striatum, ventromedial striatum, dorsolateral striatum, and ventrolateral striatum at the level of the central striatum (approximately AP, 9.2 and 8.8 from interaural level according to the atlas of Paxinos and Watson, 1986). Selected areas were observed under a light microscope (Axioscope 9500) equipped with $20 \times$ or $40 \times$ bright-field objective. The distribution of immunoreactive cells was examined and counted on each side of the brain. Data in two to four sections per structure were averaged for each rat, and the mean \pm SEM of these values were calculated for each group on each side of the brain (six to eight rats per group).

Drugs. The neurotoxin 6-OHDA hydrobromide (Sigma) was prepared in saline containing $0.1 \%$ ascorbic acid. The noradrenaline uptake inhibitor desipramine hydrochloride (Sigma) was prepared in injectable water and administered in a volume of $2 \mathrm{ml} / \mathrm{kg}$ body weight. $m$-CPP (Research Biochemicals, Milwaukee, WI) was diluted (calculated as free base) in $\mathrm{NaCl} 0.9 \%$ and injected in a volume of $1 \mathrm{ml} / \mathrm{kg}$ body weight in the case of peripheral administration. Apomorphine (Sigma) was dissolved (calculated as free base) in saline containing $0.01 \%$ ascorbic acid and injected subcutaneously in a volume of $1 \mathrm{ml} / \mathrm{kg}$ body weight. The $\mathrm{pH}$ of final solutions for all drugs and vehicle was neutral.

Statistical analysis. Oral bouts were quantified during each $10 \mathrm{~min}$ period for $60 \mathrm{~min}$, and results are expressed as the mean \pm SEM. Data were analyzed by a two-way ANOVA, considering the lesion of DA neurons (pretreatment) and $m$-CPP (treatment) as the main factors. When a significant interaction was found $(p<0.05)$, indicating that the effect of the treatment is modified by the pretreatment, this first statistical analysis was followed by a one-way ANOVA (using group as the main factor), completed by the post hoc Scheffe's test to allow adequate multiple comparisons between groups. Differences in time course of effect between groups was assessed as described above (two-way ANOVA; 6-OHDA $\times m$-CPP) on each $10 \mathrm{~min}$ data point for the $60 \mathrm{~min}$ period analysis. A separate one-way ANOVA followed by the post hoc Dunnett's test was performed to determine the effect of the various doses of $m$-CPP locally administered into the subthalamic nucleus of shamlesioned animals.

Results for Fos immunohistochemistry experiments are expressed as the mean \pm SEM of immunoreactive cells per square millimeter for each structure. Data obtained in the side ipsilateral and contralateral to the lesion were separately analyzed by a two-way ANOVA (6-OHDA $\times$ $m$-CPP), as described above. A one-way ANOVA was subsequently done to determine whether $m$-CPP and 6-OHDA lesion affected Fos expression by themselves in the various brain regions examined. All statistical analyses were done with StatView 5.0 (SAS Institute Inc., Cary, NC). In all cases, the threshold for significance was $p<0.05$.

\section{RESULTS \\ Lesion of DA neurons}

The extent of the lesion of DA neurons was assessed by (1) turning behavior after injection of apomorphine $(0.05 \mathrm{mg} / \mathrm{kg}$, s.c. $)$ and (2) immunolabeling for $\mathrm{TH}$, the rate limiting step of catecholamine synthesis, in midbrain sections. All animals retained for analysis showed $>100$ turns per $30 \mathrm{~min}$ in response to apomorphine. In these animals, immunoreactivity for TH was virtually absent on the side of the lesion along the entire rostrocaudal extent of the substantia nigra (Fig. 1). The loss of neurons in the substantia nigra pars compacta was confirmed histologically with a cresyl violet staining performed on adjacent sections (Delfs et al., 1995). Immunoreactivity for $\mathrm{TH}$ in the ventral tegmental area, a region adjacent to the substantia nigra pars compacta that contains dopaminergic cell bodies innervating cortical and limbic regions, was mostly spared by 6-OHDA in our experimental conditions (data not shown).

\section{Nigrostriatal lesions increase oral movements induced by peripheral administration of $m$-CPP}

The effect of a unilateral lesion of the nigrostriatal DA pathway on the ability of the nonselective $5-\mathrm{HT}_{2 \mathrm{C}}$ agonist $m$-CPP to elicit oral movements is shown in Figure 2. As reported previously, the systemic administration of $m$-CPP ( $1 \mathrm{mg} / \mathrm{kg}$, i.p.) stimulated oral bouts during a $1 \mathrm{hr}$ observation period (76.9 \pm 3.4 vs $25.1 \pm 4.5$ in sham-lesioned animals treated with $m$-CPP and saline, respectively, mean \pm SEM, $n=8)$. This effect was markedly increased $(+119 \%)$ in rats with a 6-OHDA-induced lesion $(139.3 \pm 5$ oral bouts per hour, mean $\pm \mathrm{SEM}, n=6$ ) (two-way ANOVA, treatment $\times$ pretreatment, $\left.F_{(1,24)}=49.9, p<0.0001\right]$. The lesion did not modify the number of oral movements by itself $(25.3 \pm 4.8$, NS, Scheffe's test, $n=6$ ). Moreover, the lesion of DA neurons did not modify the time course of effect of $m$-CPP on oral movements. Indeed, the time peak of effect was reached $30 \mathrm{~min}$ after the injection of $m$-CPP in both sham- and 6-OHDA-lesioned rats, and values slowly decreased to almost control values at the end of the experiment (data not shown). Of note, $m$-CPP did not induce any turning behavior in dopamine-depleted rats. 


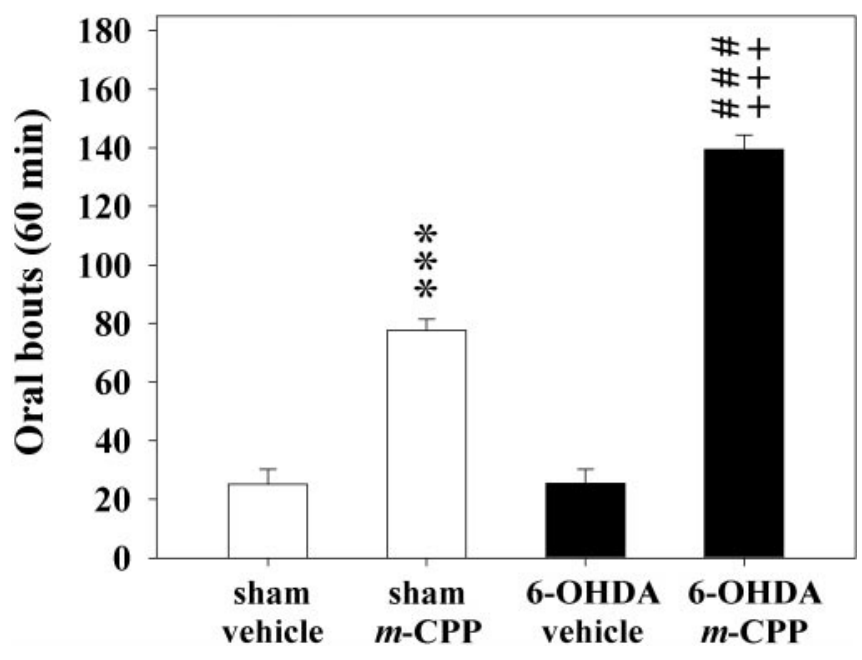

Figure 2. Effect of a unilateral lesion of DA neurons on $m$-CPP-induced oral bouts. The data represent the mean \pm SEM of oral bouts recorded for $60 \mathrm{~min}$ after drug or vehicle injection ( $n=6-8$ rats per group). Experiments were performed 4.5-5.5 weeks after the injection of 6-OHDA or its vehicle (sham) in the left substantia nigra. Each rat received only one injection of vehicle or $m$-CPP $(1 \mathrm{mg} / \mathrm{kg}$, i.p.). Lesions of dopaminergic neurons by 6-OHDA increased $m$-CPP-induced oral movements (two-way ANOVA; see Results). ${ }^{* * *} p<0.001$ versus salinetreated rats with a sham-lesion; \#\#\# $p<0.001$ versus $m$-CPP-treated rats with a sham lesion; $+++p<0.001$ versus saline-treated rats with a 6-OHDA lesion (Scheffe's test).

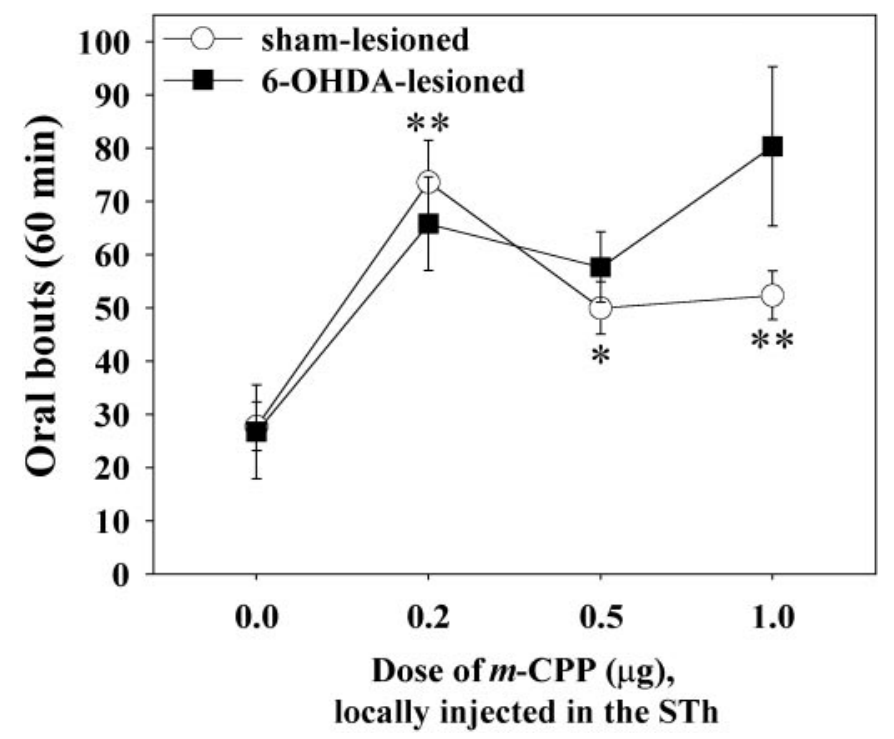

Figure 3. Effect of a unilateral lesion of nigrostriatal dopaminergic neurons on oral bouts induced by the infusion of $m$-CPP into the left subthalamic nucleus $(S T h)$. Data are expressed as the mean \pm SEM number of oral bouts per $60 \mathrm{~min}(n=6-8)$. Doses of $m$-CPP $(0,0.2,0.5$, and $1 \mu \mathrm{g}$ in $0.1 \mu \mathrm{l}$ of $\mathrm{NaCl} 0.9 \%$ ) were administered over 4 weeks in a counterbalanced order with a 1 week drug-free period between drug administrations. The nigrostriatal lesion did not modify $m$-CPP-induced oral bouts (two-way ANOVA; see Results). ${ }^{*} p<0.05$ and ${ }^{* *} p<0.01$ (compared with vehicle injection, Dunnett's test following significant one-way ANOVA, see Results).

\section{Role of subthalamic neurons in the increase in $m$-CPP-induced oral movements in rats with a dopaminergic lesion}

The effect of a unilateral injection of $m$-CPP $(0,0.2,0.5$, and $1 \mu \mathrm{g})$ into the subthalamic nucleus on oral movements in sham- and
6-OHDA-treated rats is shown in Figure 3. As reported previously for animals without lesions (Eberle-Wang et al., 1996), $m$-CPP, locally administered into the nucleus at $0.5,1$, and 0.2 $\mu \mathrm{g} / 100 \mathrm{nl}$ significantly enhanced the incidence of oral movements (one-way ANOVA, dose as the main factor, $F_{(3,24)}=11.9, p<$ $0.0001 ; n=6-8)$. The maximal effect was obtained after the administration of the lowest dose $(74 \pm 7.9$ oral bouts, mean \pm SEM, $p<0.01$, Dunnett's test) (Fig. 3). After the injection of 0.5 and $1 \mu \mathrm{g}$ of $m$-CPP, the time peak of effect was observed after 30 min, and values returned to saline control levels 50 min after the injection of the drug. In contrast, the effect induced by $0.2 \mu \mathrm{g}$ of $m$-CPP was almost stable during the period of measurement and even slightly increased with time (data not shown).

The lesion of dopaminergic neurons did not modify oral movements induced by $m$-CPP locally administered into the subthalamic nucleus, regardless of the dose administered (two-way ANOVA, treatment $\times$ pretreatment, $F_{(3,44)}=1.78, n=8$ rats, NS) (Fig. 3). Also, the time course of the effect induced by $m$-CPP was not different in 6-OHDA-treated rats than in rats with a sham lesion (data not shown).

The histological analysis did not reveal differences in subthalamic nucleus injection sites between sham- and 6-OHDA-treated rats. Indeed, the majority (six of eight for both sham- and 6-OHDA-treated rats) of tracks left by cannulas were located in the central part of the subthalamic nucleus (corresponding to plates 35-36 with respect to Paxinos and Watson, 1986). Three tracks were rostrolateral, whereas one was extremely caudal (in a 6-OHDA-treated rat). As noted previously (Eberle-Wang et al., 1996), implantations localized outside the subthalamic nucleus (removed from the statistical analysis of the data) corresponded to animals that did not develop oral movements after subthalamic injection of $m$-CPP (data not shown).

\section{Effect of $m$-CPP on Fos expression in the basal ganglia of rats with sham and 6-OHDA lesions}

\section{Striatum}

As reported previously for the indirect serotonergic agonist fenfluramine (Rouillard et al., 1996), immunoreactivity for Fos was heterogeneous in the striatum after injections of saline or $m$-CPP, with the medial striatum containing more labeled cells than the lateral striatum. Therefore, the density of Fos-positive nuclei was analyzed in four quadrants delimiting the dorsolateral, ventrolateral, dorsomedial, and ventromedial striatum (Rouillard et al., 1996; see Materials and Methods).

In the dorsomedial striatum, the number of Fos-immunoreactive nuclei $\left(51.7 \pm 14.3\right.$ cells $/ \mathrm{mm}^{2}$ on the left side, mean \pm SEM, $n=7$ sham-lesioned and vehicle-injected rats) increased to $518 \pm 74$ cells $/ \mathrm{mm}^{2}$ after $m$-CPP administration $(n=8)$ (Fig. 4$)$. The immunoreactive neurons were organized in clusters of irregular shape and size. The density of Fos-immunoreactive cells after $m$-CPP administration was strongly reduced $(-80 \%, n=6)$ by a lesion of nigrostriatal dopaminergic neurons on the same side (two-way ANOVA, treatment $\times$ pretreatment, $F_{(1,23)}=16.4, p<$ $0.0005)$ (Figs. 4, 5A-C). The lesion of dopaminergic neurons by itself did not significantly modify the control levels of Fos (76 \pm 14.8, Scheffe's test, $n=6$ ). Moreover, on the intact side, the ability of $m$-CPP to induce Fos immunoreactivity in the dorsomedial striatum (474 \pm 78 vs $51 \pm 7.2$ cells $/ \mathrm{mm}^{2}$ in sham-lesioned animals receiving $m$-CPP and saline, respectively, $n=8-7$ ) was not significantly modified in 6-OHDA-lesioned rats $(421 \pm 98$ 

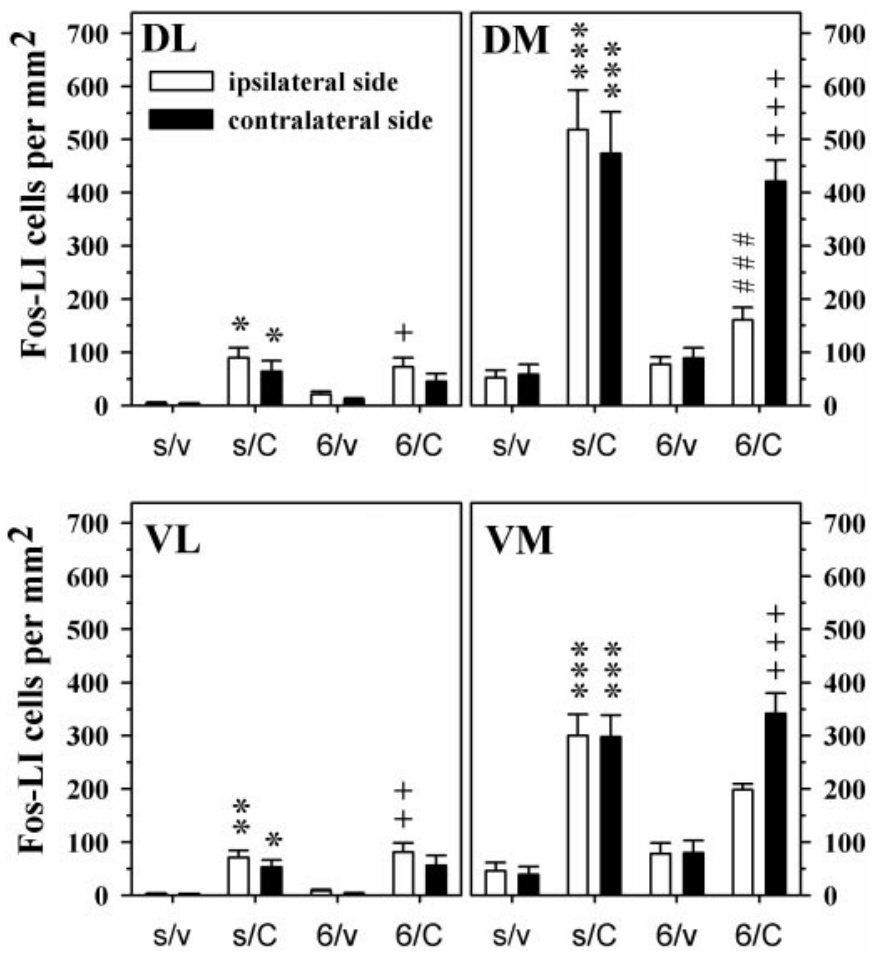

Figure 4. Quantitative analysis of the density of Fos-like immunoreactive cells in the dorsolateral $(D L)$, dorsomedial $(D M)$, ventrolateral $(V L)$, and ventromedial $(V M)$ quadrants of the striatum after systemic administration of $m$-CPP $(1 \mathrm{mg} / \mathrm{kg}$, i.p. $)$ in rats with sham- and 6-OHDAinduced lesions. The data are expressed as the mean \pm SEM of Fos-like immunoreactive cells per square millimeter on the side of the lesion (white bars) and the contralateral side (black bars) in each experimental group. $s / v$, Sham/vehicle; $s / C$, sham $/ m$-CPP; 6/v, 6-OHDA/vehicle; $6 / C$, 6-OHDA $/ m$-CPP ( $n=6-8$ animals per group). Experiments were performed 4.5-5.5 weeks after the injection of 6-OHDA or its vehicle in the left substantia nigra. The nigrostriatal lesion decreased the number of Fos-like immunoreactive cells after $m$-CPP administration on the side of the lesion only in the dorsomedial and ventromedial striatum (two-way ANOVA; see Results). ${ }^{*} p<0.05,{ }^{* *} p<0.01$, and ${ }^{* * *} p<0.001$ compared with saline-treated rats with a sham-lesion; $+p<0.05,++p<0.01$, and $+++p<0.001$ compared with saline-treated rats with a 6-OHDA lesion; $\# \# \# p<0.001$ compared with $m$-CPP-treated rats with a sham-lesion (Scheffe's test).

cells $/ \mathrm{mm}^{2}$, mean \pm SEM, $n=6$ ) (two-way ANOVA, treatment $\times$ pretreatment, $F_{(1,23)}=0.63$, NS, $\left.n=6-8\right)$.

A similar pattern of response to $m$-CPP administration was observed in the ventromedial striatum. Indeed, $m$-CPP induced an increase of Fos immunoreactivity $\left(300 \pm 40\right.$ cells $\left./ \mathrm{mm}^{2}, n=8\right)$, and this effect was significantly reduced $(-50 \%, n=5)$ on the side of the lesion in dopamine-depleted rats (two-way ANOVA, treatment $\times$ pretreatment, $\left.F_{(1,22)}=5.3, p<0.05, n=5-8\right)$. A similar enhancement of $m$-CPP-induced Fos expression was seen in the ventromedial striatum on the side contralateral to the lesion and was not affected by the lesion of dopaminergic neurons (two-way ANOVA, treatment $\times$ pretreatment, $F_{(1,22)}=0.002$, NS, $n=5-8$ ).

In the left dorsolateral striatum, $m$-CPP significantly enhanced Fos expression $\left(89 \pm 18\right.$ vs $4.3 \pm 1.2 \mathrm{cells} / \mathrm{mm}^{2}$ in sham-lesioned rats treated with $m$-CPP and saline, respectively; $n=7-8)$, an effect that was not modified by the lesion of DA neurons (two-way ANOVA, treatment $\times$ pretreatment, $F_{(1,23)}=1.5$, NS, $\left.n=6-8\right)$. Similar results were observed on the side contralateral to the lesion (two-way ANOVA, treatment $\times$ pretreatment, $F_{(1,22)}=$
0.9, NS). Similarly, the increase in Fos immunoreactivity induced by $m$-CPP in the ventrolateral striatum of rats with a sham lesion $\left(m\right.$-CPP, $71 \pm 14$ and $53 \pm 19$ immunoreactive cells $/ \mathrm{mm}^{2}$ vs saline, $2.6 \pm 0.8$ and $2.4 \pm 0.4$ immunoreactive cells $/ \mathrm{mm}^{2}$ in left and right sides, respectively) was not modified by the lesion of DA neurons (two-way ANOVA, treatment $\times$ pretreatment, $F_{(1,23)}=$ 0.03 , NS, and $F_{(1,23)}=0.005$, NS, respectively, $\left.n=6-8\right)$.

\section{Globus pallidus}

Very few Fos-immunoreactive cells were found in the globus pallidus (equivalent to the external pallidum of primates) of saline-treated rats with sham lesions $(n=7)$ (Fig. 6). Moreover, despite a trend to increase, $m$-CPP did not significantly modify Fos expression (Scheffe's test). 6-OHDA-induced lesion did not affect this outcome (two-way ANOVA, treatment $\times$ pretreatment, $F_{(1,22)}=0.013$, NS, $\left.n=6-7\right)$. The lesion of dopaminergic neurons by itself did not significantly modify basal Fos immunoreactivity (Scheffe's test). Also, no significant difference was observed on the intact side after $m$-CPP injection in rats with or without a lesion of dopaminergic neurons (two-way ANOVA, treatment $\times$ pretreatment, $\left.F_{(1,22)}=0.9, \mathrm{NS}, n=6-7\right)$.

\section{Subthalamic nucleus}

An average of $19 \pm 7$ and $20 \pm 12$ labeled cells $/ \mathrm{mm}^{2}$ (mean \pm SEM, $n=7$ ) was observed in the left and right subthalamic nucleus, respectively, of animals with sham lesions and treated with saline. The dopaminergic lesion by itself did not significantly modify Fos immunoreactivity (Scheffe's test, $n=6$ ). $m$-CPP significantly increased Fos expression in the subthalamic nucleus on both sides in rats with sham lesions ( $68 \pm 5$ and $68 \pm 10$ in the left and right sides, $p<0.01$ and $p<0.05$, respectively, Scheffe's test after one-way ANOVA, $n=8$ ) (Fig. 6). This effect was not affected by the lesion of dopaminergic neurons regardless of the side considered (two-way ANOVA, treatment $\times$ pretreatment, $F_{(1,23)}=0.03$, NS, and $F_{(1,23)}=1.2, \mathrm{NS}$, in the left and right side, respectively).

\section{Entopeduncular nucleus}

Despite a trend to increase the number of Fos-positive cells $(9.8 \pm 2.8$ vs $3.4 \pm 1.2$ in $m$-CPP- and saline-treated rats, respectively, $n=8-7$ ) (Fig. 6), $m$-CPP did not significantly modify basal Fos expression in the left entopeduncular nucleus in rats with sham lesions (Scheffe's test). However, m-CPP markedly increased Fos-positive cells in rats with 6-OHDA lesions $(41 \pm 9.7$ immunoreactive cells $/ \mathrm{mm}^{2}, n=6$ ) (two-way ANOVA, treatment $\times$ pretreatment, $\left.F_{(1,23)}=8.5, p<0.01\right)$. Immunoreactive cells were mainly localized in the middle of the rostrocaudal extent of the region and tended to occupy the lateral part of the entopeduncular nucleus. The lesion by itself did not significantly affect basal Fos expression (7.4 \pm 1.4 , Scheffe's test, $n=6)$. The slight increase in Fos immunoreactivity elicited by $m$-CPP in sham-lesioned rats reached significance on the side contralateral to the lesion $(3.16 \pm 1.2$ vs $14.1 \pm 2.6, p<0.05$, Scheffe's test, $n=$ $7-8$ ) (Fig. 6) but not in lesioned side (4.5 \pm 1.2 vs $10.4 \pm 2.3$, NS, $n=7-8$ ), and the lesion did not significantly alter $m$-CPP effects on that side (two-way ANOVA, treatment $\times$ pretreatment, $F_{(1,23)}$ $=1.98$, NS, $n=6-8)$.

\section{Substantia nigra}

As reported previously (Whirtshafter and Asin, 1995; Sgambato et al., 1997), very few Fos-immunoreactive cells were found along the rostrocaudal extent of the substantia nigra pars reticulata in 

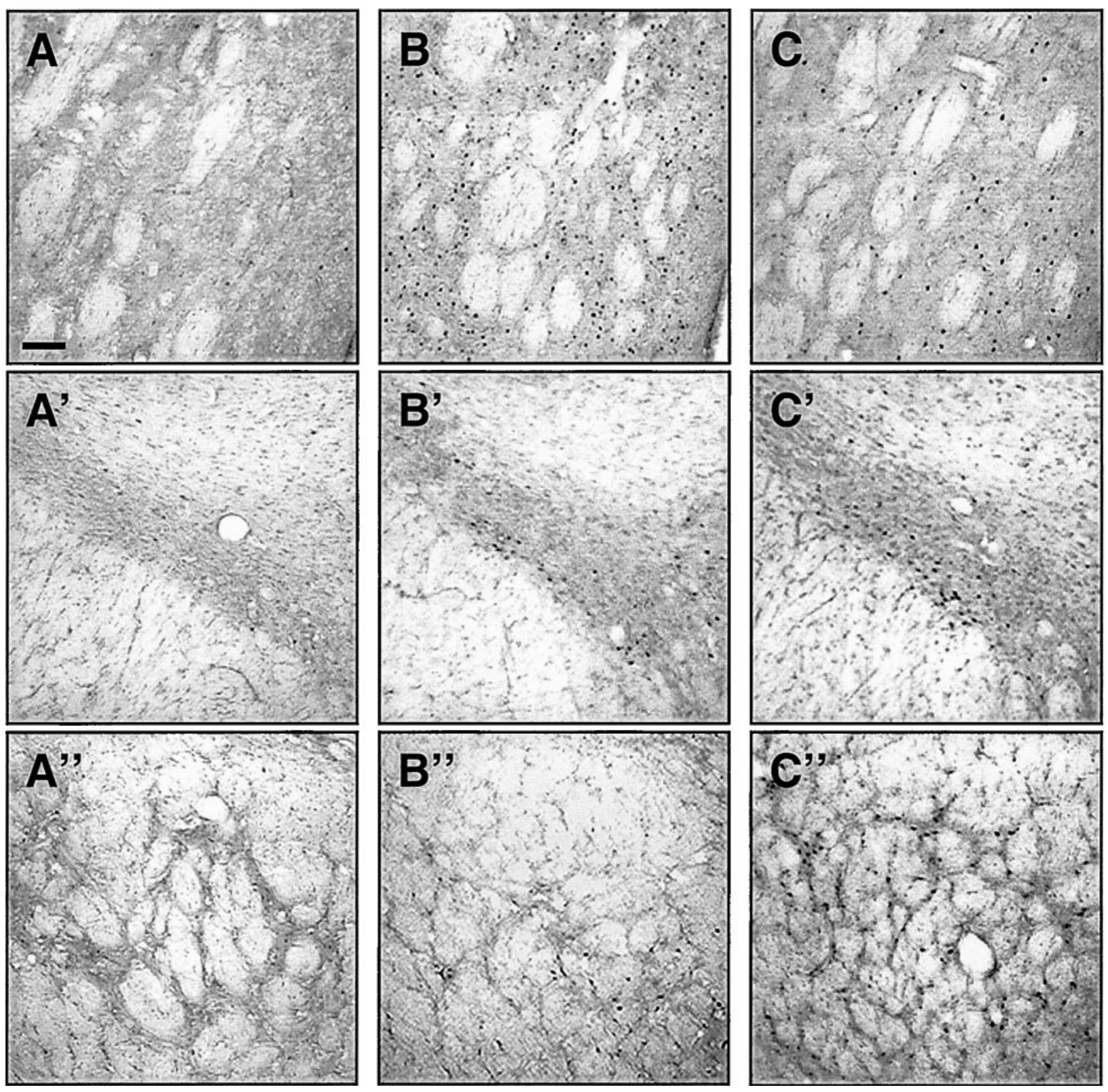

Figure 5. Photomicrographs of Fos-like immunoreactivity in the striatum (top row), subthalamic nucleus (middle row), and entopeduncular nucleus (bottom row) after administration of saline $\left(A, A^{\prime}, A^{\prime \prime}\right)$ or $m$-CPP $\left(B, B^{\prime}, B^{\prime \prime}\right)$ in rats with a sham lesion, and administration of $m$-CPP in rats with a 6-OHDA lesion $\left(C, C^{\prime}, C^{\prime \prime}\right)$. Scale bar (in $A$ ): $A-C^{\prime \prime}, 100 \mu \mathrm{m}$.

saline-treated rats with a sham lesion. The moderate effects of $m$-CPP on Fos immunoreactivity did not reach significance (Scheffe's test) (Fig. 6) and were not affected by the 6-OHDA lesion (two-way ANOVA, treatment $\times$ pretreatment, $F_{(1,24)}=$ 0.057 , and $F_{(1,24)}=0.876, n=6-8, \mathrm{NS}$, on the left and right sides, respectively).

\section{DISCUSSION}

The results of this study reveal that the unilateral destruction of nigrostriatal DA neurons in adult rats alters in vivo responses to a peripheral administration of the 5-HT agonist $m$-CPP in the basal ganglia.

\section{Increased $\boldsymbol{m}$-CPP-induced oral dyskinesia after nigrostriatal lesions}

As reported previously, peripheral administration of $m$-CPP induced an increase in oral movements (Stewart et al., 1989; Gong et al., 1992; Eberle-Wang et al., 1996). Based on previous pharmacological studies, the dose of $1 \mathrm{mg} / \mathrm{kg} \mathrm{m}$-CPP used in this study induces almost half of the maximal effect of $m$-CPP on oral movements (Gong et al., 1992; Eberle-Wang et al., 1996). A common property of 5-HT antagonists that reduce this effect is the ability to block $5-\mathrm{HT}_{2 \mathrm{C}}$ but not $5-\mathrm{HT}_{2 \mathrm{~A}}$ or $5-\mathrm{HT}_{1 \mathrm{~B}}$ receptors (Gong et al., 1992; Eberle-Wang et al., 1996). In addition, 5-HT agonists at $5-\mathrm{HT}_{1 \mathrm{~A}}$ or/and $5-\mathrm{HT}_{1 \mathrm{~B}}$ receptors do not induce oral movements (Stewart et al., 1989; Gong et al., 1992). Together, these data indicate that oral movements elicited by $m$-CPP are likely mediated by $5-\mathrm{HT}_{2 \mathrm{C}}$ receptors.

The ability of $m$-CPP to induce oral movements was dramatically enhanced by a unilateral lesion of nigral dopaminergic neurons. This observation extends previous evidence that chronic impairment of nigrostriatal DA transmission facilitates $m$-CPPinduced oral movements. Indeed, bilateral lesions of dopaminer- 

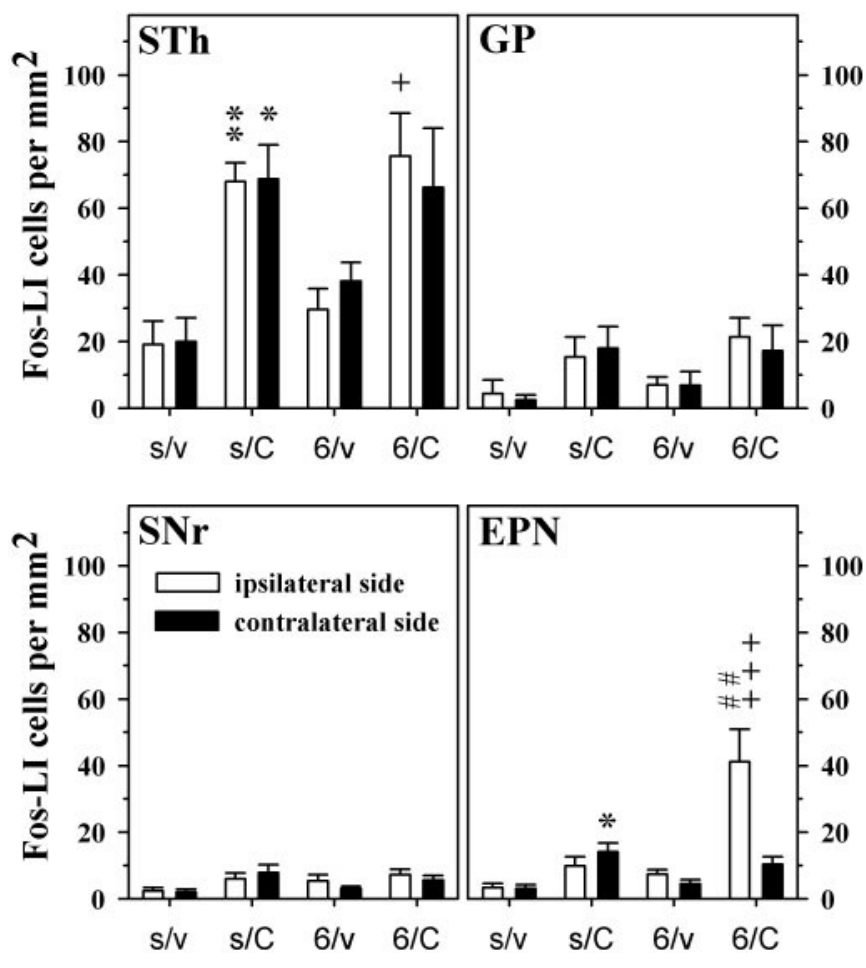

Figure 6. Quantitative analysis of the density of Fos-like immunoreactive cells in the GP, EPN, SNr, and subthalamic nucleus (STh) after systemic administration of $m$-CPP $(1 \mathrm{mg} / \mathrm{kg}$, i.p.) in rats with sham and 6-OHDA-induced lesions. The data are expressed as the mean \pm SEM of Fos-like immunoreactive cells per square millimeter on the side of the lesion (white bars) and the contralateral side (black bars) in each experimental group. $s / v$, Sham/vehicle; $s / C$, sham/m-CPP; 6/v, 6-OHDA/vehicle; $6 / C, 6-\mathrm{OHDA} / m$-CPP ( $n=6-8$ animals per group). Experiments were performed 4.5-5.5 weeks after the injection of 6-OHDA or its vehicle in the left substantia nigra. The nigrostriatal lesion significantly increased the number of Fos-like immunoreactive cells after $m$-CPP administration on the side of the lesion only in the EPN (two-way ANOVA; see Results). $m$-CPP induced by itself a significant increase of Fos-like immunoreactive cells in the subthalamic nucleus (one-way ANOVA; see Results). ${ }^{*} p<0.05$ and $* * p<0.01$ compared with salinetreated rats with a sham-lesion; $+p<0.05$ and $+++p<0.001$ compared with saline-treated rats with a 6-OHDA-lesion; \#\# $<0.01$ compared with $m$-CPP-treated rats with a sham lesion (Scheffe's test).

gic neurons in neonatal rats increased $m$-CPP-induced oral movements in adults (Gong and Kostrzewa, 1992), and chronic administration of the dopamine antagonist haloperidol potentiated $m$-CPP-induced oral movements (Schutz et al., 1998).

We have shown previously that infusion of the $5-\mathrm{HT}_{2 \mathrm{C}}$ receptor antagonists mesulergine or SDZSER082 into both subthalamic nuclei reduced dyskinesia induced by the peripheral administration of $m$-CPP, indicating that this effect involved the subthalamic nucleus (Eberle-Wang et al., 1996). However, subthalamic $5-\mathrm{HT}_{2 \mathrm{C}}$ receptors were not directly responsible for the increase in oral dyskinesia observed after peripheral $m$-CPP administration in 6-OHDA-lesioned rats. Indeed, the effect of local administration of the drug into the subthalamic nucleus was not increased after lesion. Furthermore, the ability of $m$-CPP to induce Fos expression in subthalamic neurons was not modified in rats with a 6-OHDAinduced lesion. Together, these data suggest that other sites are involved in the increased behavioral response to $m$-CPP after dopaminergic lesions.

\section{$m$-CPP-induced Fos expression in the striatum}

Our finding that $m$-CPP ( $1 \mathrm{mg} / \mathrm{kg}$, i.p.) enhances Fos expression in the striatum agrees with evidence that $m$-CPP modulates striatal cells activity (El Mansari et al., 1994) and with previous data showing an increase in the number of Fos-immunoreactive striatal cells after higher doses $(5 \mathrm{mg} / \mathrm{kg}$ ) of the same drug (Cook and Wirtshafter, 1995). The 5-HT $1 \mathrm{~B} / 2 \mathrm{C}$ agonists $m$-trifluoromethylphenylpiperazine and RU-24969, the 5- $\mathrm{HT}_{2 \mathrm{~A} / 2 \mathrm{C}}$ agonist 2,5-dimethoxy-4-iodoamphetamine or the 5-HT releasers fenfluramine and $p$-chloroamphetamine have also been shown to enhance the number of Fos-immunoreactive cells in the striatum (Leslie et al., 1993; Moorman and Leslie, 1996; Rouillard et al., 1996; Cook and Whirtshafter, 1998).

Interestingly, striatal Fos expression induced by $m$-CPP was reduced by the lesion of nigrostriatal DA neurons in the dorsomedial and ventromedial quadrants. The smaller effect of dopaminergic lesion observed in the ventromedial compared with the dorsomedial region could be attributable to the sparing of the DA input from the ventral tegmental area (Gerfen et al., 1987) in our experimental conditions. Fenfluramine-induced increase in Fosimmunoreactive cells was also reduced in the striatum by a lesion of DA neurons and by the DA-D ${ }_{1}$ antagonist SCH-23390 (Rouillard et al., 1996). In the case of fenfluramine, it is possible that the lesion exerted its effect by preventing an increase in striatal DA release (De Deurwaerdère et al., 1995). However, such mechanism is unlikely to play a role after $m$-CPP injections because this drug does not affect striatal DA release at the dose used in this study (Di Giovanni et al., 2000).

Our results and that of these previous studies clearly indicate that the ability of $m$-CPP to induce Fos in the striatum is, at least in part, dependent on the tonic control exerted by DA on striatal neurons. Whereas $5-\mathrm{HT}_{2 \mathrm{C}}$ receptor stimulation has been clearly shown to account for oral movements induced by $m$-CPP in rats with or without neonatal dopaminergic lesions (Gong and Kostrzewa, 1992; Eberle-Wang et al., 1996), the ability of $5 \mathrm{mg} / \mathrm{kg}$ $m$-CPP to enhance the number of Fos-immunoreactive cells in the striatum was not reduced by the $5-\mathrm{HT}_{2 \mathrm{~A} / 2 \mathrm{C}}$ antagonist ritanserin (Cook and Whirtshafter, 1995). Despite the high dose used by these authors, likely recruiting several mechanisms (Kahn and Wetzler, 1991; Eriksson et al., 1999), it is possible that Fos expression after $m$-CPP administration is not solely mediated by $5-\mathrm{HT}_{2 \mathrm{C}}$ receptors. In the absence of selective $5-\mathrm{HT}_{2 \mathrm{C}}$ agonists and antagonists, extensive pharmacological characterizations will be necessary to identify the 5-HT receptors involved in this effect. Whatever the receptor(s) involved, the altered effect of $m$-CPP in dopamine-depleted rats could be attributable to a change either in the receptors themselves or in signal transduction mechanisms linking the receptor to Fos expression. Of note, striatal $5-\mathrm{HT}_{2 \mathrm{C}}$ receptor mRNA levels were not modified in adult rats with a lesion of the nigrostriatal dopaminergic neurons (Numan et al., 1995).

\section{Alterations in $\boldsymbol{m}$-CPP-induced Fos expression in the entopeduncular nucleus after dopaminergic lesions}

The only other region of the basal ganglia that showed a difference in $m$-CPP-induced Fos expression after nigrostriatal lesion is the entopeduncular nucleus. The mechanism of the marked increase in Fos induction observed in this region after nigrostriatal lesions is unclear. In primates, dopaminergic lesions increase the firing rate of neurons of the internal pallidum, a region equivalent to the entopeduncular nucleus, and modify their firing pattern (DeLong, 1990; Brotchie et al., 1991; Boraud et al., 1998). 
Similar cellular changes in rats could enhance the response to the stimulation of $5-\mathrm{HT}_{2 \mathrm{C}}$ receptors that are present in this region (Pazos et al., 1985; Eberle-Wang et al., 1997). The effects of $5-\mathrm{HT}_{2 \mathrm{C}}$ agonists on entopeduncular neurons are, however, not known. Striatal GABAergic neurons that project to the entopeduncular nucleus could also participate in this effect. Indeed, previous data showed a reduction of fenfluramine-induced Fos immunoreactivity in these neurons by D1 antagonists (Rouillard et al., 1996). This suggests that dopamine may facilitate a serotonergic regulation of these neurons. Thus, in the absence of dopamine, $m$-CPP would be unable to regulate (presumably activate) this inhibitory input to the entopeduncular nucleus, unmasking a direct effect of $m$-CPP on Fos expression in this region.

Interestingly, a similar increase in Fos expression was not observed in the substantia nigra pars reticulata. A difference in distribution of $5-\mathrm{HT}_{2 \mathrm{C}}$ receptors between entopeduncular nucleus and substantia nigra pars reticulata could account for the differential effects of $m$-CPP in these two regions. Indeed, although functional $5-\mathrm{HT}_{2 \mathrm{C}}$ receptors have been also found in the substantia nigra pars reticulata (Rick et al., 1995; Fox et al., 1998), only a subpopulation of neurons in its caudal region express $5-\mathrm{HT}_{2 \mathrm{C}}$ receptor mRNA in contrast to virtually all neurons of the entopeduncular nucleus (Eberle-Wang et al., 1997). A differential distribution of receptors is also likely to account for the lack of robust Fos induction in the globus pallidus after $m$-CPP injections in rats with or without lesions. Indeed, neurons of the globus pallidus do not express $5-\mathrm{HT}_{2 \mathrm{C}}$ receptor mRNA, and the density of $5-\mathrm{HT}_{2 \mathrm{C}}$ receptor binding sites in this region is very low (Pazos et al., 1985; Eberle-Wang et al., 1997; Pasqualetti et al., 1999). Together, the data reveal that $m$-CPP increases Fos expression preferentially in basal ganglia subregions displaying the greatest density of $5-\mathrm{HT}_{2 \mathrm{C}}$ receptor.

\section{Functional implications}

It is unclear whether the regionally specific changes in $m$-CPPinduced Fos expression observed after nigrostriatal lesion are, in any way, related to the enhanced behavioral response observed in the same conditions. However, it is of interest to note that the two regions of the basal ganglia with lesion-induced changes in Fos expression after dopaminergic lesions have also been implicated in oral dyskinesia. The ventral striatum is involved in the control of oral activity (Salamone et al., 1990). A role for the striatum is supported by data showing that oral movements induced by a local injection of $m$-CPP into the ventral striatum are enhanced in rats treated with 6-OHDA as neonate (Plech et al., 1995). A role of the entopeduncular nucleus in dyskinesia is suggested by the antidyskinetic effect of lesions or high-frequency stimulation of its equivalent, the posteroventral region of the internal pallidum (Dogali et al., 1995; Sutton et al., 1995; Lang et al., 1999).

Hyperkinetic side effects remain a major complication of longterm treatments with neuroleptics or L-DOPA in schizophrenia or Parkinson's disease, respectively (Tarsy and Baldessarini, 1984; Chase et al., 1994). The results of the present study indicate that a lesion of dopaminergic neurons similar to that causing Parkinson's disease alters behavioral and cellular responses elicited by stimulation of 5-HT receptors. Dopaminergic agonists and L-DOPA can increase the release of 5-HT in several brain regions (Ng et al., 1972; Ferré et al., 1994; Thorre et al., 1998). Therefore, an excess of serotonergic transmission, together with the changes in functional responses to serotonin induced by the dopaminergic lesion, may be involved in the development of dyskinesia after dopamine replacement therapy. This is supported by clinical evidence that $5-\mathrm{HT}_{2 \mathrm{C}}$ antagonists can be useful to treat the complications of antiparkinsonian therapy (Ikeguchi and Kuroda, 1995; Pact and Giduz, 1999).

\section{REFERENCES}

Azmitia EC, Segal M (1978) An autoradiographic analysis of the differential ascending projections of the dorsal and median raphe nuclei in the rat. J Comp Neurol 179:641-668.

Barnes NM, Sharp T (1999) A review of central 5-HT receptors and their function. Neuropharmacology 38:1083-1152.

Boraud T, Bezard E, Guehl D, Bioulac B, Gross C (1998) Effects of L-DOPA on neuronal activity of the globus pallidus externalis (GPe) and globus pallidus internalis (GPi) in the MPTP-treated monkey. Brain Res 787:157-160.

Brotchie P, Iansek R, Horne MK (1991) Motor function of the monkey globus pallidus. II. Cognitive aspects of movement and phasic neuronal activity. Brain 114:1685-1670.

Chase TN, Engber TM, Mouradian MM (1994) Palliative and prophylactic benefits of continuously administered dopaminomimetics in Parkinson disease. Neurology 44:S15-S18.

Chesselet M-F (2000) Dopamine-GABA interactions In: Handbook of experimental pharmacology. (Di Chiara G, ed). Heidelberg: Springer.

Clemett DA, Punhani T, Duxon MS, Blackburn TP, Fone KCF (2000) Immunohistochemical localisation of the $5-\mathrm{HT}_{2 \mathrm{C}}$ receptor protein in the rat CNS Neuropharmacology 39:123-132.

Cook DF, Wirtshafter D (1995) Serotonin agonist-induced c-fos expression in the rat striatum. Soc Neurosci Abstr 21:1424.

Cook DF, Wirtshafter D (1998) Quinpirole attenuates striatal c-fos induction by 5-HT, opioid and muscarinic receptor agonists. Eur J Pharmacol 349:41-47.

De Deurwaerdère $\mathrm{P}$, Spampinato U (1999) Role of 5-HT $2 \mathrm{~A}$ and 5- $\mathrm{HT}_{2 \mathrm{C}}$ receptor subtypes in the control of accumbal and striatal dopamine release elicited in vivo by dorsal raphe nucleus electrical stimulation. J Neurochem 73:1033-1042.

De Deurwaerdère P, Bonhomme N, Le Moal M, Spampinato U (1995) D-Fenfluramine increases striatal extracellular dopamine in vivo independently of serotonergic terminals or dopamine uptake sites. J Neurochem 65:1100-1108.

Delfs JM, Ciaramitaro VM, Parry T, Chesselet M-F (1995) Subthalamic nucleus lesions: widespread effects on changes in gene expression induced by nigrostriatal dopamine depletion in rats. J Neurosci 15:6562-6575.

DeLong MR (1990) Primate models of movement disorders of basal ganglia origin. Trends Neurosci 13:281-285.

Di Giovanni G, Di Matteo V, Di Mascio M, Esposito E (2000) Preferential modulation of mesolimbic vs. nigrostriatal dopaminergic function by serotonin ${ }_{2 \mathrm{C} / 2 \mathrm{~B}}$ receptor agonists: a combined in vivo electrophysiological and microdialysis study. Synapse 35:53-61.

Dogali M, Fazzani E, Kolodny E, Eidelberg D, Sterio D, Devinski O, Beric A (1995) Stereotaxic ventral pallidotomy for Parkinson's disease. Neurology 45:753-761.

Ebabi M, Srinivasan SK (1995) Pathogenesis, prevention, and treatment of neuroleptic-induced movement disorders. Pharmacol Rev 47:575-604.

Eberle-Wang K, Lucki I, Chesselet M-F (1996) A role for the subthalamic nucleus in $5-\mathrm{HT}_{2 \mathrm{C}}$-induced oral dyskinesia. Neuroscience 72:117-128.

Eberle-Wang K, Mikeladze Z, Uryu K, Chesselet M-F (1997) Pattern of expression of the Serotonin ${ }_{2 \mathrm{C}}$ receptor messenger RNA in the basal ganglia of adults rats. J Comp Neurol 384:233-247.

El Mansari M, Radja F, Ferron A, Reader TA, Molina-Holgado E, Descarries L (1994) Hypersensivity to serotonin and its agonists in serotonin-hyperinnervated neostriatum after neonatal dopamine denervation. Eur J Pharmacol 261:171-178.

Eriksson E, Engberg G, Bing O, Nissbrandt H (1999) Effects of mCPP on the extracellular concentrations of serotonin and dopamine in rat brain. Neuropsychopharmacology 20:287-296.

Ferré S, Cortés R, Artigas F (1994) Dopaminergic regulation of the serotonergic raphe-striatal pathway: microdialysis studies in freely moving rats. J Neurosci 14:4839-4846.

Fox S, Moser B, Brotchie J (1998) Behavioral effects of 5- $\mathrm{HT}_{2 \mathrm{C}}$ receptor antagonism in the substantia nigra pars reticulata of the 6-hydroxydopamine-lesioned rat model of Parkinson's disease. Exp Neurol 151:35-49. 
Gerfen CR, Herkenham M, Thibault J (1987) The neostriatal mosaïc. II. Patch-and matrix-directed mesostriatal dopaminergic and nondopaminergic systems. J Neurosci 7:3915-3934.

Gong L, Kostrzewa RM (1992) Supersensitized oral response to a serotonin agonist in neonatal 6-OHDA-treated rats. Pharmacol Biochem Behav 41:621-623.

Gong L, Kostrzewa RM, Fuller RW, Perry KW (1992) Supersensitization of oral response to SKF 38393 in neonatal 6-OHDA-lesioned rats is mediated through a serotonine system. J Pharmacol Exp Ther 261:1000-1007.

Ikeguchi K, Kuroda A (1995) Mianserin treatment of patients with psychosis induced by antiparkinsonian drugs. Eur Arch Psychiatry Clin Neurosci 244:320-324.

Kahn RS, Wetzler S (1991) m-Chlorophenylpiperazine as a probe of serotonin receptors. Biological Psychiatry 30:1139-1166.

Kostrzewa RM, Reader TA, Descarries L (1998) Serotonin neural adaptations to ontogenic loss dopamine neurons in rat brain. J Neurochem 70:889-898.

Lang AE, Duff J, Saint-Cyr JA, Trepanier L, Gross RE, Lombardi W, Montgomery E, Hutchinson W, Lozano AM (1999) Posteroventral pallidotomy in Parkinson's disease. J Neurol 246:II/28-II/41.

Leslie RA, Moorman JM, Coulson A, Grahame-Smith DG (1993) Serotonin2/1C receptor activation causes a localized expression of the immediate-early gene c-fos in rat brain: evidence for involvement of dorsal raphe nucleus projection fibres. Neuroscience 53:457-463.

Mehta A, Thermos K, Chesselet M-F (2000) Increased behavioral response to dopaminergic stimulation of the subthalamic nucleus after nigrostriatal lesions. Synapse, in press.

Melamed E, Zoldan J, Friedberg G, Ziv I, Weizmann A (1996) Involvement of serotonin in clinical features of Parkinson's disease and complications of L-DOPA therapy. Adv Neurol 69:545-550.

Meltzer HY, Nash JF (1991) Effects of antipsychotic drugs on serotonin receptors. Pharmacol Rev 43:587-604.

Moorman JM, Leslie RA (1996) $p$-Chloroamphetamine induces $c$-fos in rat brain: a study of serotonin $2 \mathrm{~A} / 2 \mathrm{C}$ receptor function. Neuroscience 72:129-139.

Ng LKY, Chase TN, Colburn RW, Kopin IJ (1972) L-DOPA in parkinsonism: a possible mechanism of action. Neurology 22:688-696.

Numan S, Lundgren KH, Wright DE, Herman JP, Seroogy KB (1995) Increased expression of 5-HT2 receptor mRNA in rat striatum following 6-OHDA lesions of the adult nigrostriatal pathway. Mol Brain Res 29:391-396.

Pact V, Giduz T (1999) Mirtazapine treats resting tremor, essential tremor, and levodopa-induced dyskinesias. Neurology 53:1154.

Parry TJ, Eberle- Wang K, Lucki I, Chesselet M-F (1994) Dopaminergic stimulation of subthalamic nucleus elicits oral dyskinesia in rats. Exp Neurol 128:181-190.

Pasqualetti M, Ori M, Castagna M, Marazziti D, Cassano GB, Nardi I
(1999) Distribution and cellular localization of the serotonin type 2C receptor messenger RNA in human brain. Neuroscience 92:601-611.

Paxinos G, Watson C (1986) The rat brain in stereotaxic coordinates, Ed 2. Sydney: Academic.

Pazos A, Cortés R, Palacios JM (1985) Quantitative autoradiographic mapping of serotonin receptors in the rat brain. II. Serotonin-2 receptors. Brain Res 346:231-249.

Plech A, Brus R, Kalbfleisch JH, Kostrzewa RM (1995) Enhanced oral activity responses to intrastriatal SKF 38393 and m-CCP are attenuated by intrastriatal mianserin in neonatal 6-OHDA-lesionned rats. Psychopharmacology 119:466-473.

Rick CE, Stanford IM, Lacey MG (1995) Excitation of rat substantia nigra pars reticulata neurons by 5-hydroxytryptamine in vitro: evidence for a direct action mediated by 5 -hydroxytryptamine $2 \mathrm{C}$ receptors. Neuroscience 69:903-913.

Roth BL, Ciaranello RD, Meltzer HY (1992) Binding of typical and atypical antipsychotic agents to transiently expressed 5-HT1C receptors. J Pharmacol Exp Ther 260:1361-1365.

Rouillard C, Bovetto S, Gervais J, Richard D (1996) Fenfluramineinduced activation of the immediate-early gene c-fos in the striatum: possible interaction between serotonin and dopamine. Mol Brain Res $37: 105-115$

Salamone JD, Johnson CJ, McCullough LD, Steinpreis RE (1990) Lateral striatal cholinergic mechanisms involved in oral motor activities in the rat. Psychopharmacology 85:71-79.

Schutz LJ, Moore DJ, Wolf WA (1998) Haloperidol increases serotonin (5-HT) $5-\mathrm{HT}_{2 \mathrm{C}}$ receptor function in caudate. 28th Annual Meeting of Society for Neuroscience, Los Angeles, CA, November.

Sgambato V, Abo V, Rogard M, Besson MJ, Deniau JM (1997) Effect of electrical stimulation of the cerebral cortex on the expression of the Fos protein in the basal ganglia. Neuroscience 81:93-112.

Stewart BR, Jenner P, Marsden CD (1989) Induction of purposeless chewing behavior in rats by 5-HT agonists drugs. Eur J Pharmacol 162:101-107.

Sutton JP, Coudwell W, Lew MF, Mallory L, Grafton S, DeGiorgio C, Welsh M, Apuzzo MLJ, Ahmadi J, Waters CH (1995) Ventroposterior medial pallidotomy in patients with advanced Parkinson's disease. Neurosurgery 36:1112-1117.

Tarsy D, Baldessarini RJ (1984) Tardive dyskinesia. Annu Rev Med 35:605-623.

Thorre K, Sarre S, Smolders I, Ebinger G, Michotte Y (1998) Dopaminergic regulation of serotonin release in the substantia nigra of the freely moving rat using microdialysis. Brain Res 796:107-116.

Trent F, Tepper JM (1991) Dorsal raphé stimulation modifies striatalevoked antidromic invasion of nigral dopaminergic neurons in vivo. Exp Brain Res 84:620-630.

Wirtshafter D, Asin KE (1995) Dopamine antagonists induce fos-likeimmunoreactivity in the substantia nigra and entopeduncular nucleus of the rat. Brain Res 670:205-214. 